\title{
Genomic and transcriptomic analyses of the Chinese Maotai-flavored liquor yeast MT1 revealed its unique multi-carbon co-utilization
}

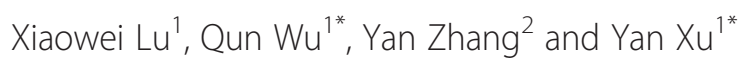

\begin{abstract}
Background: Revealing genetic mechanisms behind specific physiological characteristics of Saccharomyces cerevisiae from specific environments is important for industrial applications and requires precise understanding.

Results: Maotai strain MT1 was isolated from the complicated Chinese Maotai-flavored liquor-making environment with extremely high temperatures, and acidic and ethanol stresses. Compared with the type strain S288c, MT1 can tolerate high acidity ( $\mathrm{pH}$ 2.0), high ethanol levels (16\%) and high temperatures $\left(44^{\circ} \mathrm{C}\right)$. In addition, MT1 can simultaneously utilize various sugars, including glucose, sucrose, galactose, maltose, melibiose, trehalose, raffinose and turanose. Genomic comparisons identified a distinct MT1 genome, $0.5 \mathrm{Mb}$ smaller than that of S288c. There are 145 MT1-specific genes that are not in S288c, including MEL1, MAL63, KHR1, BIO1 and BIO6. A transcriptional comparison indicated that HXT5 and HXT13, which are theoretically repressed by glucose, were no longer inhibited in MT1 and were highly expressed even in a medium containing $70 \mathrm{~g} / \mathrm{L}$ glucose. Thus, other sugars may be co-utilized with glucose by MT1 without diauxic growth.
\end{abstract}

Conclusions: Based on a functional genomics analysis, we revealed the genetic basis and evolutionary mechanisms underlying the traits of the Chinese Maotai-flavored yeast MT1. This work provides new insights for the genetic breeding of yeast and also enriches the genetic resources of yeast.

Keywords: Saccharomyces cerevisiae, Genome, Transcriptome, Maotai-flavour liquor

\section{Background}

The Chinese liquor-making process is a unique and complicated spontaneous fermentation process under solid state conditions [1]. Chinese liquor is produced by the technique of simultaneous saccharification and fermentation (SSF) using grains, such as sorghum, wheat and barley. The cost-effective SSF combines the enzymatic hydrolysis of starch with the simultaneous fermentation of sugars, resulting in an outpouring carbon source [2]. In addition, Chinese liquor is fermented using combinations of microorganisms, including yeast, bacteria and

\footnotetext{
*Correspondence: wuqun_1981@163.com; yxu@jiangnan.edu.cn

'State Key Laboratory of Food Science and Technology; The Key Laboratory

of Industrial Biotechnology, Ministry of Education; Synergetic Innovation

Center of Food Safety and Nutrition; School of Biotechnology, Jiangnan

University, Wuxi, Jiangsu, China

Full list of author information is available at the end of the article
}

filamentous fungi [3]. The complex filamentous fungal community produces diversified hydrolases to degrade starch into various sugars, including glucose, galactose, maltose and melibiose [4]. High temperatures $\left(\sim 50{ }^{\circ} \mathrm{C}\right)$ at the starter are generated by the metabolic activities of the microorganisms and the low thermal conductivities of the solid matrices [5]. In addition, there are two other prominent pressures: acidity stress $(\mathrm{pH} 3.0)$ and ethanol stress $(4.5-5.5 \%, \mathrm{v} / \mathrm{v})$ in the alcoholic fermentation stage [6]. Such a severe environment during the SSF process leads to a distinctive community of microorganisms with specific physiological properties and performances [7], in particular, distinctive heatand acid-resistant properties [8].

Although the microorganisms in the Chinese Maotaiflavored liquor have been investigated since the 1960s



(c) 2015 Lu et al. Open Access This article is distributed under the terms of the Creative Commons Attribution 4.0 International License (http://creativecommons.org/licenses/by/4.0/), which permits unrestricted use, distribution, and reproduction in any medium, provided you give appropriate credit to the original author(s) and the source, provide a link to the Creative Commons license, and indicate if changes were made. The Creative Commons Public Domain Dedication waiver (http:// creativecommons.org/publicdomain/zero/1.0/) applies to the data made available in this article, unless otherwise stated. 
[9], there is little information on the functional genomics of the yeast involved in the process. This has inhibited our understanding of the fermentation mechanism and the development of Maotai-flavored liquor. Functional genomics approaches, such as whole-genome sequencing, are powerful tools for the analysis of specific traits on a genomic scale and provide a method of studying cellular physiology from micro-perspective. Since the first complete genome of strain S288c was sequenced in 1996 [10], it has served as the reference for the Saccharomyces cerevisiae species and has promoted the development of whole-genome approaches [11]. Meanwhile, the genomes of several $S$. cerevisiae strains have been sequenced and compared with the S288c reference genome, including laboratory strains [12] and industrial strains, such as sake yeast K7, wine yeasts, beer yeasts and bioethanol yeasts [13-17]. The phenotypic diversity among yeast isolates is significant, and variation is apparent among the surveyed strains at different levels. Ecological factors and geographical locations, as the main reasons for biodiversity, have important effects on the domestication of microbial physiological characteristics [18]. Among the 50 strains with sequenced genomes in the NCBI database, there are only three strains from China [19]. However, strains involved in the SSF process of Chinese liquor are poorly understood. Theoretically, the genomic analysis of strains with different backgrounds should help identify the sequence changes that play important roles in specific physiological characteristics. Comparisons of the publicly available $S$. cerevisiae genome sequences have revealed the clear signatures [single nucleotide polymorphisms (SNPs), insertions and deletions (Indels), and novel ORFs] in the different strains [20]. Compared with laboratory strains, industrial strains generally show a higher adaptability to specific environments. However, the genetic basis for their improved characteristics is not well understood. Further studies are needed to explore how the genetic variations confer the specific phenotypes. The comprehensive identification of polymorphisms among individuals within a species is essential for studying the genetic basis of phenotypic differences and for elucidating the evolutionary history of the species.

In this study, S. cerevisiae MT1 was isolated from the fermentation environment of Chinese Maotai-flavored liquor. Its physiological characteristics were evaluated in several phenotypic assays. Meanwhile, the MT1 genome was sequenced and compared with the type strain S288c. In addition, we elucidated how genetic variations of MT1 were correlated with specified traits using RNAseq and revealed the molecular mechanisms related to the adaptation of yeast in Chinese liquor-making. This whole-genome analysis of the $S$. cerevisiae strain used in Chinese liquor-making will supplement the genomic and phylogenetic knowledge of yeast and provide a guide for the construction of strains with desired traits.

\section{Results and discussion \\ Phenotypic characterization}

Several physiological and biochemical characteristics of the $S$. cerevisiae strain MT1, isolated during the process Chinese Maotai-flavored liquor-making, were compared with those of the reference strain S288c and other industrial strains (Fig. 1a). In addition to the sake yeast K7, other strains had high tolerance to $38{ }^{\circ} \mathrm{C}$ which is consistent with previous studies that it was widely believed that S. cerevisiae had a thermal tolerance of $25-37^{\circ} \mathrm{C}$ or $39{ }^{\circ} \mathrm{C}$ [21]. While a higher thermal resistance was found for MT1 when temperature was higher than $38{ }^{\circ} \mathrm{C}$ compared with not only S288c but also other industrial yeasts. Population of MT1 was twice that of other strains (besides the sake yeast K7) in $16 \%$ ethanol. It indicated that MT1 had a higher ethanol tolerance. In addition, MT1 had a growth advantage compared with other strains at $\mathrm{pH}$ 3.5-2.0. In particular, the population of MT1 is almost three times of that of S288c at $\mathrm{pH}$ 2.0. This suggested that MT1 has a much higher resistance to acidity than S288c and other industrial strains, as most industrial strains could barely grow at $\mathrm{pH} 2.5$ [22].

Carbon source utilization exerts a strong influence on the classification of isolates and may be a useful and functional measure for the characterization and classification of different strains. Unfortunately, there is limited research on the simultaneous fermentation of various carbon sources to produce ethanol. The primary reason is that not many strains can ferment ethanol from various sugars. Presently, there is some research on a strain that could simultaneous utilize glucose and xylose, or arabinose and galactose [23]. MT1s ability to uptake and assimilate sugars was also investigated (Fig. 1b). Among the monosaccharides, glucose could be used for growth and fermentation by both MT1 and S288c, with $\mathrm{OD}_{590}$ and $\mathrm{OD}_{730}$ values exceeding 0.8 . When galactose was the sole carbon source, the $\mathrm{OD}_{590}$ and $\mathrm{OD}_{730}$ of MT1 reached 1.23 and 0.98 , respectively, while those of S288c were less than 0.3. This indicated that MT1 could uniquely utilize galactose. The other carbon sources that MT1 could uniquely uptake and assimilate were mainly disaccharides, such as melibiose, maltose, trehalose and turhanose. In addition, raffinose, a trisaccharide constituted by galactose, fructose and glucose, as well as methyla-D-glucopyranoside, are well utilized by MT1, with $\mathrm{OD}_{730}$ values reaching 1.15 and 0.95 , respectively. These results suggested that MT1 was a strain appropriate for multi-carbon fermentation, having the ability to utilize various carbon sources. 


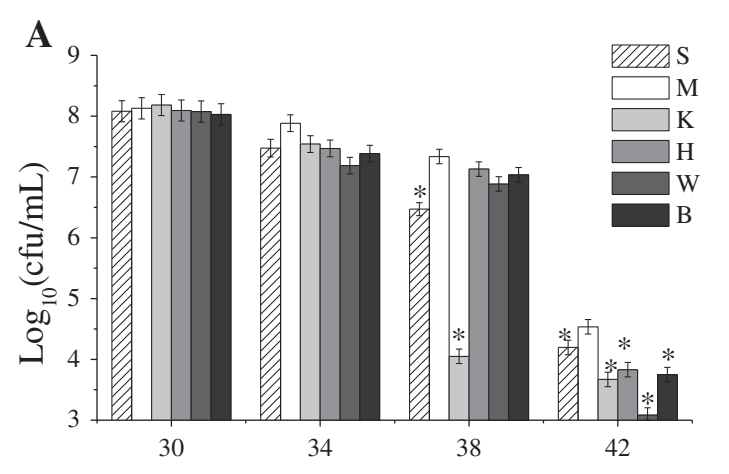

Temperature $\left({ }^{\circ} \mathrm{C}\right)$
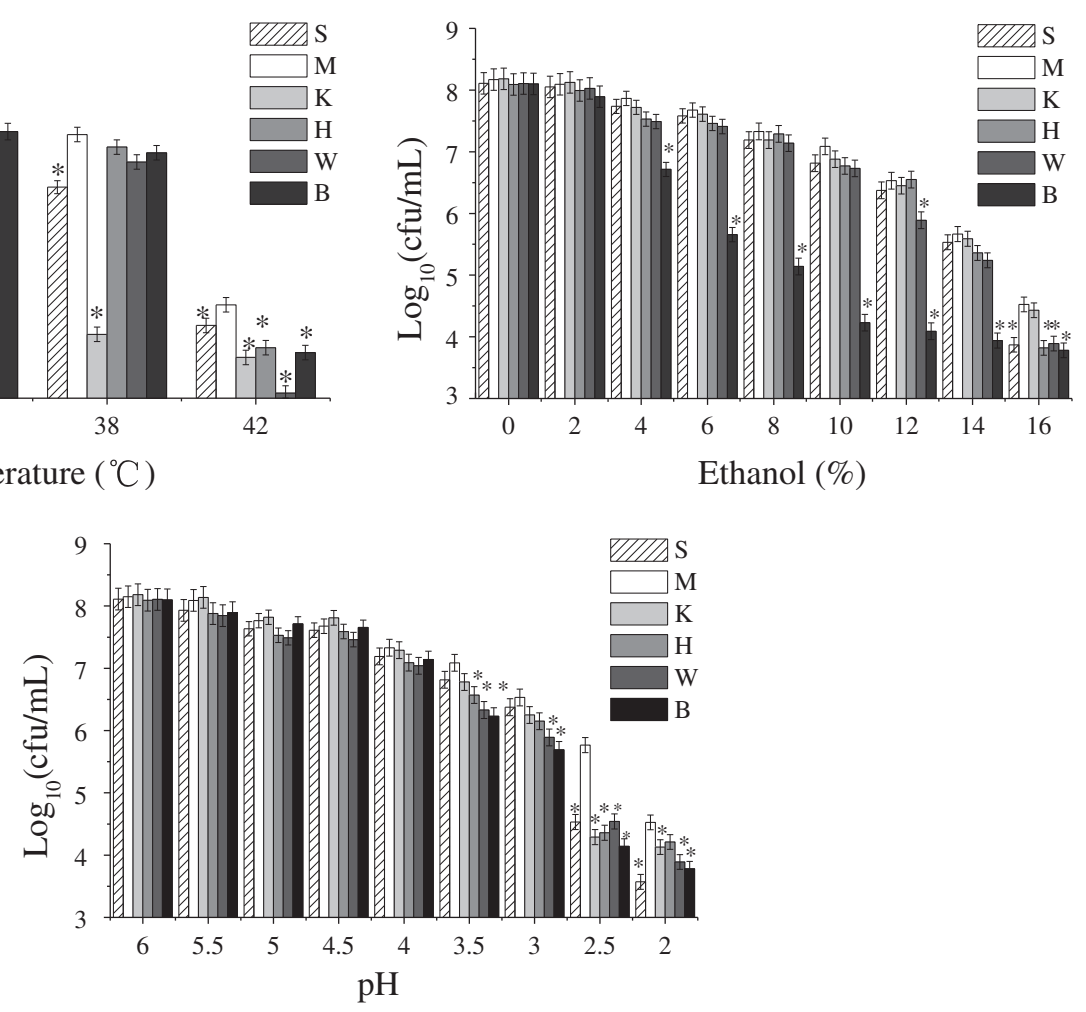

B
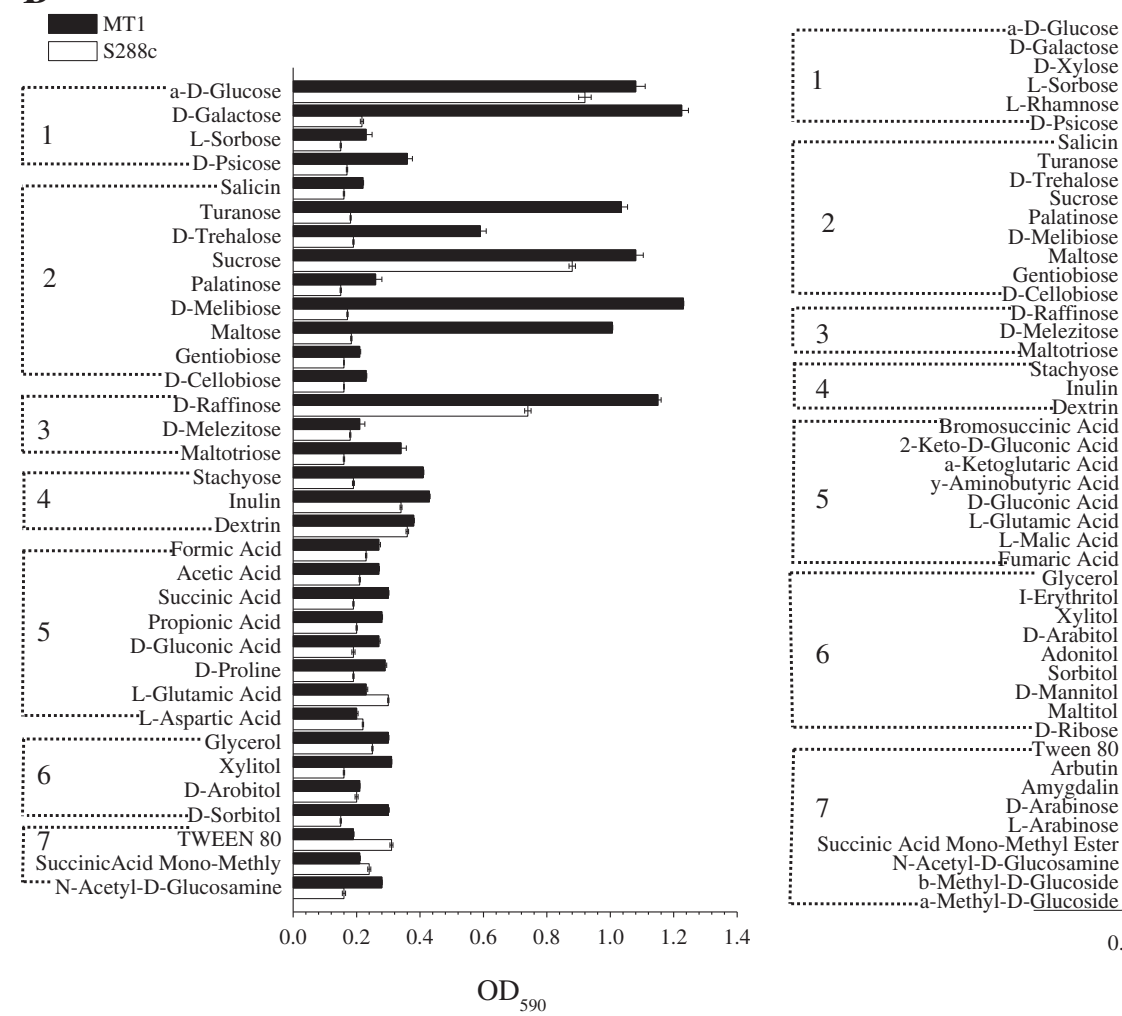

1 D-Xylose L-Rhamnose

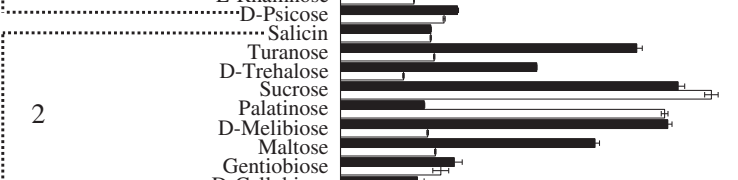
Gentiobiose



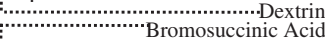

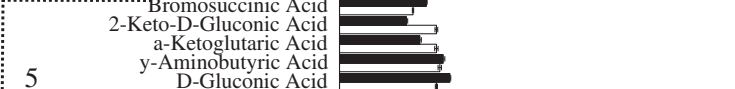

$5 \quad \mathrm{y}$-Amintoric

L-Glutamic Acid

L-Malic Acid
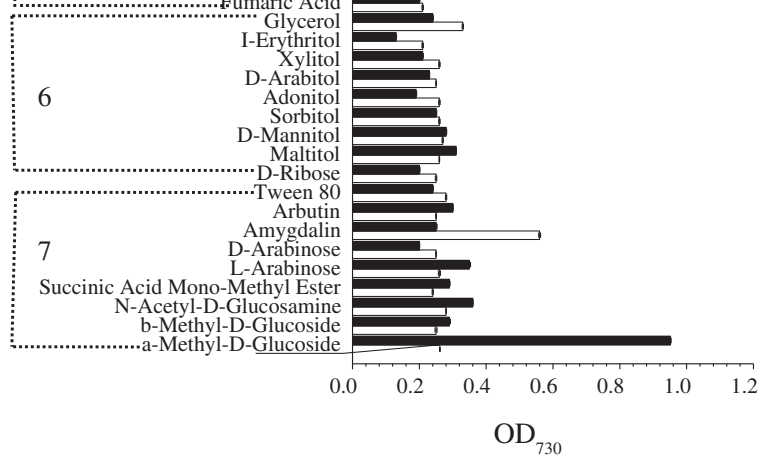

Fig. 1 (See legend on next page.) 
(See figure on previous page.)

Fig. 1 a Effects of temperature, ethanol and acidity on cell growth of several yest strains. S, Saccharomyces cerevisiae type strain S288c; M, Chinese Maotai-flavored liquor yeast MT1; K, Sake yeast strain K7; H, Hangjiu (Chinese Rice Wine) yeast CICC30394; W, Wine yeast strain CICC31089; B, Beer yeast strain ATCC9763. ${ }^{*}$ represents the significant difference with $P$-value $<0.01$, compared with MT1, calculated using a one-way analysis of variance in SPSS. b Growth differences between MT1 and S288c with various carbon sources. Left: oxidation tests that detect carbon utilization via a redox color change in the organism's suspension; Right: assimilation reaction tests that are based on turbidity increases due to carbon utilization of the organisms. 1, monosaccharides; (2), disaccharides; (3), trisaccharides; (4), oligosaccharides; (5), acids; (6), alcohols; and (7), others

\section{MT1 genome structure}

The MT1 genome assembly resulted in 125 contigs (>200 bp) with an N50 value of 390,837. Then, 89 scaffolds (>500 bp) were obtained with an N50 value of 550,408 . The assembled data were evaluated, and the detailed statistical data are shown in Table 1. Finally, the MT1 assembled genome was $11.62 \mathrm{Mbp}$ with a GC\% of $38.08 \%$. The genome size of MT1 is smaller than most of the $S$. cerevisiae genomes deposited in the NCBI database (Table 2). A total of 5,106 genes were predicted with an average length of $1.6 \mathrm{~kb}$, occupying $69.26 \%$ of the whole genome (Table 2).

Within the aligned regions of the MT1 and S288C genomes, we identified 58,960 high-confidence SNPs $(44,847$ homozygous SNPs and 14,113 heterozygous SNPs, Additional file 1: Table S1), including 13,780 missense variants and 25,724 synonymous variants (Additional file 1: Table S2). Remarkably, the level of nucleotide polymorphism observed between MT1 and S288c is very similar to that of YJM789 $(60,339)$, a strain of $S$. cerevisiae isolated from the lung of an AIDS patient, and the wine yeast strain AWRI1631 (56,703) [15], far higher than the biofuel producing industrial strain YJS329 $(39,098)$ which was also isolated from China [19] and the commercial wine yeast strain EC1118 (46,152) [16], but far

Table 1 Chinese Maotai-flavored liquor yeast MT1 library assembly statistics

\begin{tabular}{lll}
\hline & Contigs (>200 bp) & Scaffolds (>500 bp) \\
\hline Min sequence length (bp) & 205 & 508 \\
Max sequence length (bp) & 899,603 & $1,037,495$ \\
Total sequence number & 125 & 89 \\
N20 & 749,375 & 805,328 \\
N50 & 390,837 & 550,408 \\
N90 & 128,295 & 233,221 \\
N number & 0 & 26946 \\
N rate \% & 0 & $0.23 \%$ \\
Total sequence length (bp) & $11,620,879$ & $11,620,879$ \\
GC content \% & $38.08 \%$ & $38.08 \%$ \\
Sequences greater than $1 \mathrm{~kb}$ & 73 & 44
\end{tabular}

$\mathrm{N} 20$ and N90 were obtained in the same way

${ }^{\mathrm{a}} \mathrm{N} 50$ : computed by sorting all contigs from largest to smallest and by determining the minimum set of contigs whose sizes total $50 \%$ of the entire genome lower than that of the sake yeast strain K7 $(67,725)$ [13], and the biofuel producing industrial strain JAY291 $(65,000)$ [17]. In addition, there were 6,474 small Indels ( $<90 \mathrm{bp}$ ), including 4,457 homozygous Indels and 2,017 heterozygous Indels (Additional file 1: Table S1). Within the annotated Indels, there were 251 frameshift variants that resulted in feature elongation, and 262 frameshift variants that resulted in feature truncation (Additional file 1: Table S3). The densities of SNPs and Indels were far from constant across either the whole genome (Additional file 1: Table S1) or individual chromosomes (Fig. 2a). This phenomenon mostly appears in genomes of industrial strains, such as the genome of K7, AWRI1631 and YJS329. It is more likely that a complex history of out- and/or back-crossings of the ancestral strain in the industrial environments by environmental domestication and artificial breeding could have caused this pattern. In addition, 183 copy number variations were obtained using CNVnator [24]. They were comprised of 30 duplication regions and 153 deletion regions (Additional file 1: Table S4). All of these results indicated that MT1 had a genome distinct from that of S288c. Aimed at detecting the evolutionary origin of MT1, a neighbor-joining tree was constructed on the basis of whole genome and protein sequences. The tree included MT1 and 18 other representative $S$. cerevisiae strains from library, clinic and sake, bioethanol, wine and beer production. As shown in Fig. 2b, MT1 is located far from the wine and beer strain clusters but adjacent to the sake yeast strain $\mathrm{K} 7$, and they were both isolated in Asia.

Among the 5,106 genes of MT1, less than 500 genes displayed similarity levels below $95 \%$ when compared with S288c (Additional file 1: Figure S1). Using an all-vsall BLASTP and clustering analysis with orthomcl (V2.0.8), 145 MT1-specific genes were identified, $90 \%$ of which had near-perfect-match hits to the nucleotide sequence of S288c, and likely reflect ORF annotation differences, whereas others had nonreciprocal-best-hit homologs in S288c. The functions of these genes fell within stress response (14 genes), carbohydrate metabolic process (seven genes), organic substance transport (five genes), biosynthetic process (24 genes), organic substance metabolic process (42 genes), and others (Fig. 2c). In addition, 695 genes were missing from MT1 compared with S288c. Among them, there were 25 genes and 21 genes in the location of centromere and telomeres, 
Table 2 Essential Saccharomyces cerevisiae genomic information

\begin{tabular}{|c|c|c|c|c|c|}
\hline & MT1 & S288c & K7 & AWRI1631 & YJM789 \\
\hline Genome size (Mb) & 11,62 & 12,16 & 12,39 & 11,18 & 11,99 \\
\hline GC content (\%) & 38.08 & 38.15 & 38.30 & 38.20 & 38.10 \\
\hline GC content in mRNA region (\%) & 39.62 & / & / & / & / \\
\hline rRNA number & 5 & 10 & 1 & / & / \\
\hline tRNA number & 312 & 299 & 296 & / & / \\
\hline Coding sequence number & 5,106 & 5,906 & 5,816 & 5,451 & 5,912 \\
\hline Average coding sequence length (bp) & 1,576 & 1,369 & / & / & / \\
\hline Total coding sequence length (Mb) & 8,05 & 9,16 & / & / & / \\
\hline Coding sequence of genome (\%) & 69.26 & 75.37 & / & / & / \\
\hline
\end{tabular}

respectively, where assemble errors happen frequently. These genes are Mic17p, VMA9, TAF11, TIR4, TIR2, PRP18, COX16, COX17, SHU1, MNN1, RFA1, PUF4, AVT1, TEN1, GAT3, CMS1, GAT3, SIN3, PFA4, MCD1, PSF1, PDR3, RER1, SGF29, PAU24, PAU12, PAU14, PAU1, PAU18, PAU6, PAU21, PAU22, PAU13, PAU9, PAU3, PAU15, PAU16, PAU20, PAU10, RDS1, HSP32, YPS5, AIF1, BDS1 and ENB1. $60 \%$ of 695 missing genes were hypothetical or uncharacterized ORFs encoding putative proteins of unknown functions as reported in the Saccharomyces Genome Database. The other $40 \%$ of the genes were mainly involved in ion transport, regulation of transcription and translation, DNA and RNA binding and cellular component synthesis, such as membrane components, organelle organization and cellular protein components (Fig. 2d). While there were only about 10 genes listed in S288c as essential, including $D A D 2, D A D 3$, DAD4, BRR6, RRP14, ESF2, FAP7, USO1, MCD1 and $S P C 19$. Function of each genes was listed in Additional file 1: Table S5. As indicated by the functional classification of the specific and lost genes in MT1, there is a speculation that MT1 may have a special genomic system. It weakened transcriptional, translational and cytoplasmic synthesis. On the other hand, it strengthened carbohydrate transport and metabolism, energy metabolism and informational processing (especially environmental informational processing) metabolism.

\section{Transcriptomic comparison using RNA-seq}

The transcriptomic comparison between MT1 and S288c was carried out in sorghum extract ( $\mathrm{pH}$ 6.0), which is the raw material for liquor-making. The expression levels of genes related to carbohydrate and energy metabolism were significantly up-regulated in MT1 compared with in S288c (Table 3). Within the carbohydrate metabolism, the most significant responsive pathway was the tricarboxylic acid cycle (ko00020) with 12 up-regulated genes. In addition, five genes in the pentose phosphate pathway (ko00030) and four genes in galactose metabolism (ko00052) were more highly expressed than in S288c. In energy metabolism, oxidative phosphorylation (ko00190) had the largest number of up-regulated genes. Nucleotide metabolism, translation, and cell growth and death were the responsive pathways that had a large number of down-regulated genes compared with in S288c (Table 2). Ribosome (ko03010) had the largest number of markedly lower expressed genes (84 genes). Based on the analyses of the transcriptome, we predicted that, compared with other yeast strains, MT1 tends to make higher investments in carbon and energy metabolism than transcription and translation. This is consistent with the corresponding speculation indicated by the functional classification of the specific and lost genes in MT1.

Although there was no difference between the growth of MT1 and S288c at pH 6.0, MT1 had a gradually increasing growth advantage as the $\mathrm{pH}$ decreased to 2.0 (Fig. 1a). S288c could not grow when the $\mathrm{pH}$ was below 2.5 , thus a pH level of 2.7 was selected as the control condition, versus $\mathrm{pH} 6.0$ for transcriptomic comparisons, to identify the genetic mechanisms of acid tolerance. Then, the two strains were compared at $\mathrm{pH}$ levels between 6.0 and 2.7. When the $\mathrm{pH}$ was reduced to 2.7 from 6.0, S288c genes in seven pathways were upregulated (Fig. 3a), including nucleotide metabolism (four genes), amino acid metabolism (four genes), translation (one gene) and folding, sorting and degradation (one gene). Within energy metabolism in S288c, three genes were up-regulated, while two genes were downregulated. In addition, five carbohydrate metabolism genes were down-regulated. However, there were 15 responsive pathways in the MT1 transcriptome, indicating that MT1 had a stronger response to acid than S288c. The seven responsive pathways in S288c were also identified in MT1 but involved many more responsive genes. In particular, the translational pathway in MT1 had 71 up-regulated genes. The other responsive pathways that had up-regulated genes in MT1 were involved in transcription, replication and repair, 


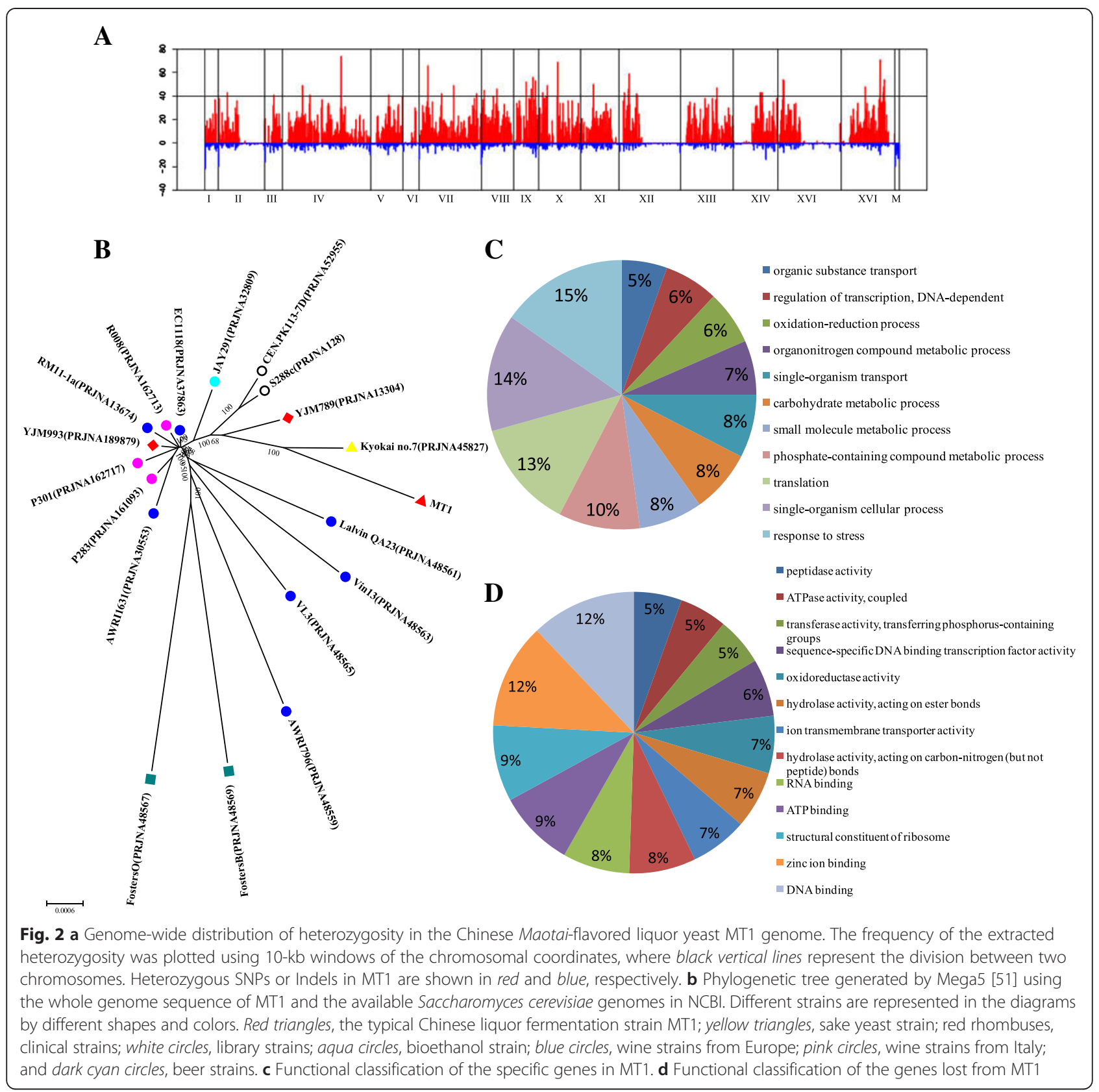

environmental information processing, transport and catabolism, cell growth and death, and cell communication (Fig. 3b). In addition, several metabolic pathways of MT1 had genes up-regulated, such as terpenoid and polyketide metabolism, xenobiotic biodegradation and metabolism, and carbohydrate metabolism. In particular, terpenoids and polyketides are the important healthy active materials in Chinese liquor. As indicated by the transcriptomic comparison, the transcription- and translation-related genes of MT1, which were inactive compared with those of S288c under normal conditions, were more active under acidic condition and achieved the same level of expression as in S288c.

Under normal conditions (sorghum juice medium, $\mathrm{pH}$ 6.0), there were not only less genes related to transcription and translation in MT1 but they were also expressed at far lower levels than in S288c; however, MT1 could attain a greater biomass than S288c. When the $\mathrm{pH}$ was reduced from 6.0 to 2.7 , genes with functions related to transcription, replication and repair and, especially, translation in MT1 were strongly up-regulated. Within these, several genes are essential to acid $\mathrm{pH}$ 
Table 3 Main differences in the metabolic pathways of the Chinese Maotai-flavored liquor yeast MT1 compared with the Saccharomyces cerevisiae type strain S288c when grown in Sorghum juice medium, pH 6.0

\begin{tabular}{|c|c|c|c|c|}
\hline Metabolism & -Log10 (P-value) & Pathway & Up genes & Down genes \\
\hline \multirow[t]{4}{*}{ Carbohydrate metabolism } & 16.82 & Tricarboxylic acid cycle cycle (ko00020) & 12 & 1 \\
\hline & & Pentose phosphate pathway (ko00030) & 5 & 3 \\
\hline & & Galactose metabolism (ko00052) & 4 & 2 \\
\hline & & Butanoate metabolism (ko00650) & 3 & 1 \\
\hline \multirow[t]{4}{*}{ Energy metabolism } & 8.34 & Oxidative phosphorylation (ko00190) & 18 & 1 \\
\hline & & Sulfur metabolism (ko00920) & 2 & 0 \\
\hline & & Carbon fixation pathways (ko00720) & 3 & 1 \\
\hline & & Sulfur metabolism (ko00920) & 2 & 0 \\
\hline \multirow[t]{2}{*}{ Nucleotide Metabolism } & 1.97 & Purine metabolism (ko00230) & 0 & 16 \\
\hline & & Pyrimidine metabolism (ko00240) & 1 & 13 \\
\hline \multirow[t]{5}{*}{ Translation } & 29.25 & Ribosome (ko03010) & 0 & 84 \\
\hline & & Aminoacyl-tRNA biosynthesis (ko00970) & 0 & 9 \\
\hline & & RNA transport (ko03013) & 0 & 9 \\
\hline & & Ribosome biogenesis in eukaryotes (ko03008) & 0 & 25 \\
\hline & & RNA degradation (ko03018) & 0 & 6 \\
\hline \multirow[t]{3}{*}{ Cell growth and death } & 1.70 & Cell cycle (ko04110) & 0 & 6 \\
\hline & & Cell cycle - yeast (ko04111) & 0 & 21 \\
\hline & & Meiosis - yeast (ko04113) & 4 & 16 \\
\hline
\end{tabular}

resistance, such as CIK1, HSP30, RPS27B, RPL31A, RPL18 and $A R O 2$. There may be two reasons for this strange phenomenon. One is that MT1 requires less proteins and nucleotides for growth than S288c. The other may be that genes involved in transcription, translation, replication and repair in MT1 are more efficient than those in S288c. There is no need to mobilize a large number of related genes under suitable conditions. Once threatened, the cell will increase its investment to survive.

\section{Genetic mechanism of multi-carbon co-utilization}

MT1 can uniquely assimilate several disaccharides, such as melibiose and maltose, and some monosaccharides, such as galactose. However, the MT1 and S288c contain
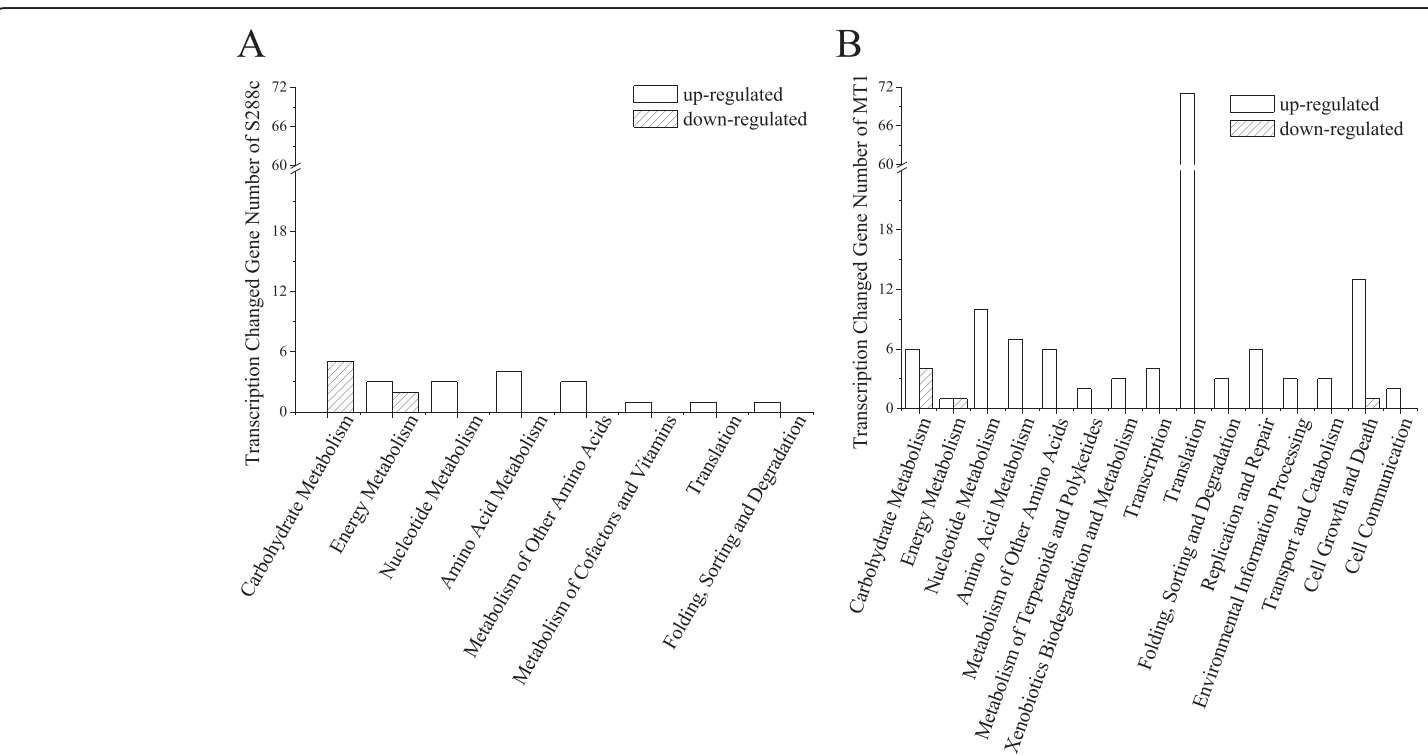

Fig. 3 Transcriptionally changed pathways of the Saccharomyces cerevisiae type strain S288c (a) and the Chinese Maotai-flavored liquor yeast MT1 (b) when the $\mathrm{pH}$ of the sorghum juice medium was reduced to 2.7 from 6.0 
A

(1)
GAL2
GAL1
GAL7
GAL5
Permease
Kinase
Transferase
Mutase

Galactose out $\longrightarrow$ Galactose in

$\rightarrow$ Gal-1-P

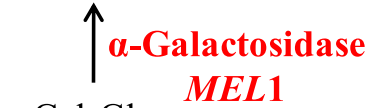

Gal-Glu

(Meliblose)

(2)

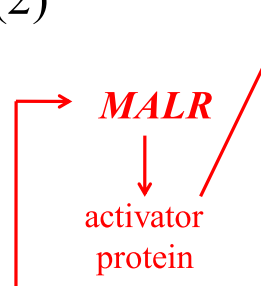

UDP-Glu

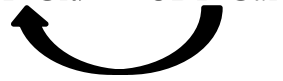

Eplmerase

GAL10

$\rightarrow$ Glu-6-P

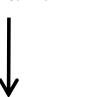

Glycolysis

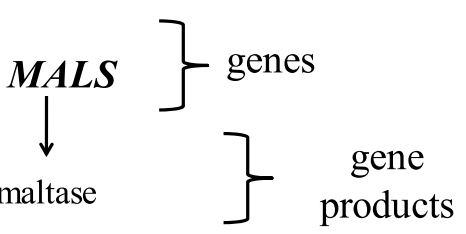

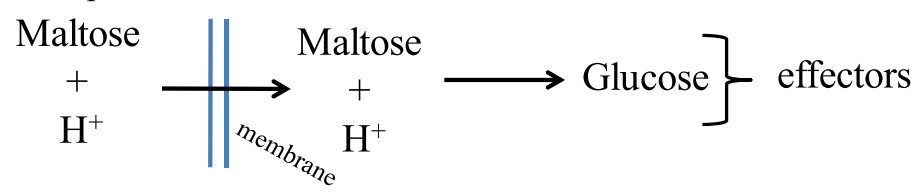

B

(1)
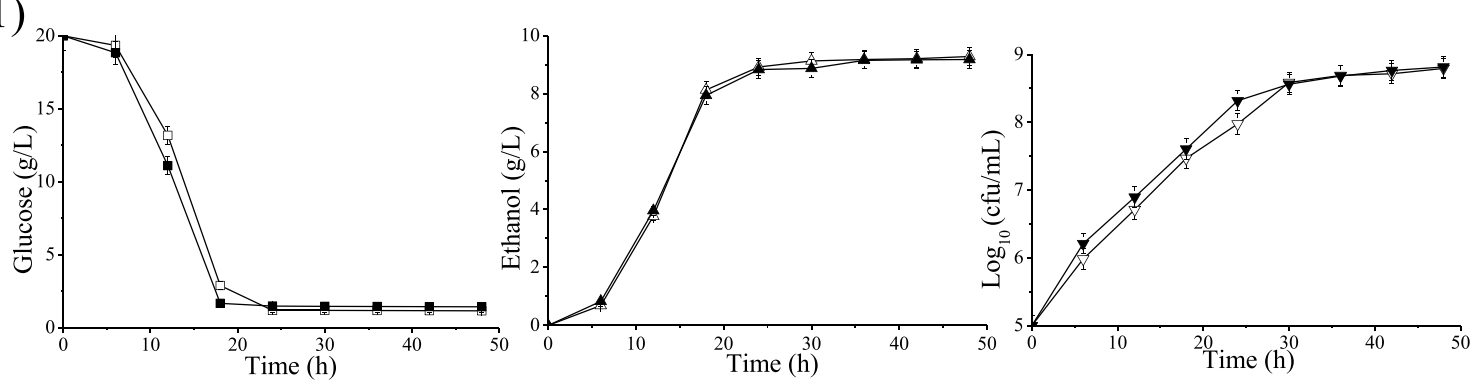

(2)

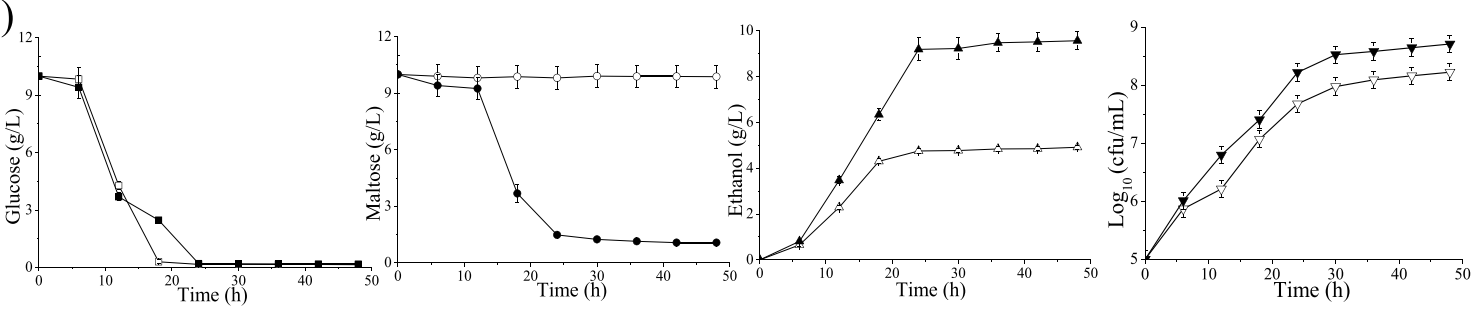

(3)

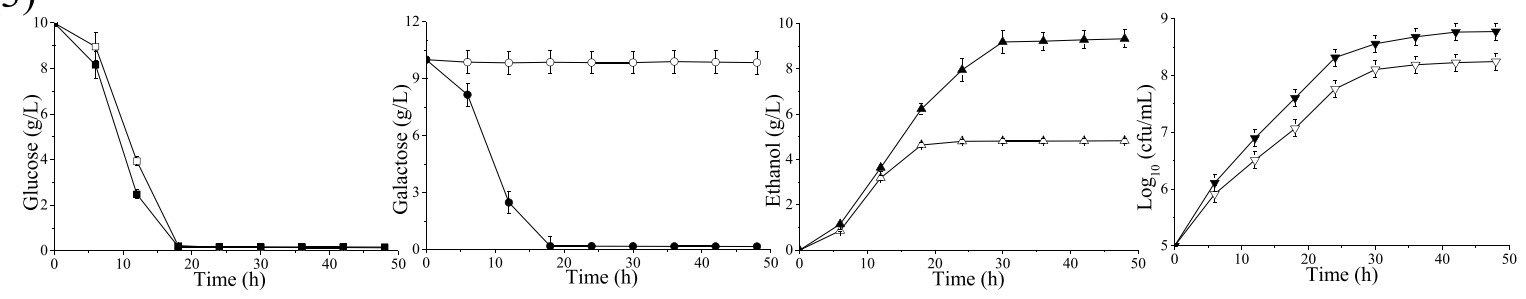

Fig. 4 (See legend on next page.) 
(See figure on previous page.)

Fig. 4 a Metabolic pathways and gene regulatory mechanisms of galactose, melibiose and maltose. The red parts of the pathways are specific to the Chinese Maotai-flavored liquor yeast MT1 strain. b Dynamic curves of fermentation parameters. (1) YPD medium (1 \% yeast extract, $2 \%$ peptone and $2 \%$ glucose); (2) modified YPD medium (1 \% yeast extract, $2 \%$ peptone, $1 \%$ glucose and $1 \%$ maltose); (3) modified YPD medium (10 \% yeast extract, $20 \%$ peptone, $1 \%$ glucose and $1 \%$ galactose). The open figures represent the Saccharomyces cerevisiae type strain S288c; the solid figures represent MT1

the same genes related to galactose utilization and its regulatory mechanism, including GAL2, GAL1, GAL7, GAL5, GAL10 and others (Fig. 4a). Therefore, a transcriptomic comparison was carried out between the two strains. It was discovered that several genes involved in galactose metabolism, such as YHR104w, YIL162W, IMA5 and others, in MT1 were up-regulated compared with those in S288c. The enzyme of YHR104w, $a k r 1$ (EC:1.1.1.21), is responsible for the transformation between D-galactose and galactitol, while YIL162W encodes the enzyme sacA (EC:3.2.1.26) which is involved in the degradation of raffinose into melibiose or galactose. The metabolic networks of melibiose and galactose are only different in the initial step, the intracellular breakdown of melibiose into glucose and galactose [25]. This step involves an alpha-galactosidase encoded by MEL1. This important gene was identified in contig 8 of MT1 but does not exist in S288c. It is most likely the reason for MT1's specific ability to utilize melibiose. This gene was identified in the genome of the laboratory strain, S. cerevisiae CEN.PK113-7D [26], a model strain for industrial biotechnology. The enzyme system of maltose is a typical inducible system in yeast cells, which includes the maltose permease and maltase [27]. The MAL loci is an important gene in maltose metabolism and is composed of three genes: MALS, MALT and MALR (Fig. 4a). The MALR genes encode maltose fermentation regulatory proteins that induce the expression of MALS, encoding the maltase (alpha-D-glucosidase), and MALT, encoding a high-affinity maltose transporter, maltose permease [28]. These three kinds of genes were identified in the MT1 genome. The two MALT genes (MAL11 and MAL31) and one MALS gene (MAL32) also exist and are functional in the genomic reference strain S288c. The differences mainly exist in the regulatory protein encoding genes: MAL13, MAL33 and MAL63. Notably, MAL63 is absent in the reference strain S288c, while MAL13 and MAL33 exist but are non-functional. In addition, there were no differences in the expression levels of MAL13 and MAL33 between MT1 and S288c. This suggests that MAL63 might be the important gene of the MT1's maltose metabolism pathway. In contrast to S288c, strains from the CEN.PK lineage are able to grow on maltose when it is the sole carbon source. However, unlike MT1, the MAL23 mutant allele known as MAL2-8C is responsible for the utilization of maltose in CEN.PK strains [26].
The transport of sugars across the cell membrane is important for growth and fermentation. Our analyses suggest that MT1 possesses different sugar uptake and assimilation properties than S288c. MT1 contains 17 HXT family genes, encoding hexose transporter proteins. Among these genes, HXT4, HXT7, HXT9, HXT12, HXT13, HXT16 and HXT17 in the MT1 genome were identified by local BLAST searches and confirmed by PCR testing. The products of these genes show different glucose affinities and different expression levels to coordinately control glucose uptake in environments containing a broad range of glucose concentrations [29]. When cells were collected for a transcriptomic analysis, we found that the expression levels of HXT5 and HXT13 in MT1 were much higher than in S288c, even though the glucose concentration in the sorghum extract adopted for the transcriptomic assay reached $70 \mathrm{~g} / \mathrm{L}$ (Table 4). This differs from a conventional report [30] that suggested that the two genes were repressed by high levels of glucose. In particular, as the glucose concentration rose from 0.2 to $40 \%$, the expression level of $H X T 13$ decreased by $80 \%$. The high expression levels of HXT5 and HXT13 indicate that MT1 may have no glucose repression and is able to simultaneously utilize multiple sugars.

To verify the multi-carbon co-utilization without glucose repression, which was indicated by the genomic and transcriptomic analyses, cell growth, sugar consumption and ethanol production were compared between MT1 and S288c in media containing mixed sugars. As shown in Fig. 4b, when compared with S288c in $2 \%$ glucose, MT1 grew faster but showed no difference in the glucose consumption and ethanol production. However, in $1 \%$ glucose plus $1 \%$ maltose, MT1 could use maltose to grow and ferment, and consequently its biomass and ethanol yield were twice that of S288c. This was also true in $1 \%$ glucose plus $1 \%$ galactose. As indicated by the dynamic curves of sugar consumption (Fig. 4b), MT1 could utilize maltose and galactose in the early stages of the logarithmic phase without the phenomenon of secondary growth. This was confirmed by the lack of a "lag" period in the growth of MT1 in a medium containing mixed sugars (Fig. 4b). These results indicate that the high expression levels of HXT5 and HXT13 without glucose repression may contribute to the multi-carbon co-utilization. 
Table 4 Comparison of the expression levels of HXT genes between the Chinese Maotai-flavored liquor yeast MT1 and the Saccharomyces cerevisiae type strain S288c when grown in sorghum juice medium (70 g/L glucose)

\begin{tabular}{|c|c|c|c|c|}
\hline Gene name & Gene function & BaseMeanMT1 & BaseMeanS288c & $P$-value \\
\hline$\overline{H X T 1}$ & $\begin{array}{l}\text { Low-affinity glucose transporter induced by Hxk2p in the presence of glucose and } \\
\text { repressed by Rgt1p when glucose is limiting. }\end{array}$ & 18.18 & 238.50 & $1.11 \times 10^{-7}$ \\
\hline HXT2 & High-affinity glucose transporter induced in low glucose and repressed in high glucose. & 2523.13 & 73238.62 & $2.66 \times 10^{-4}$ \\
\hline HXT 3 & Low affinity glucose transporter induced in low or high glucose conditions. & 770.75 & 186705.11 & $2.02 \times 10^{-7}$ \\
\hline HXT5 & $\begin{array}{l}\text { Hexose transporter with moderate affinity for glucose induced in the presence of } \\
\text { non-fermentable carbon sources and induced by a decrease in growth rate. }\end{array}$ & 191488.64 & 67393.35 & $1.96 \times 10^{-1}$ \\
\hline HXT8 & $\begin{array}{l}\text { Protein of unknown function similar to hexose transporters, expression is induced in } \\
\text { low glucose and repressed in high glucose. }\end{array}$ & 20.60 & 62.17 & $4.64 \times 10^{-1}$ \\
\hline HXT9 & $\begin{array}{l}\text { Putative hexose transporter similar to major facilitator superfamily (MFS) transporters, } \\
\text { expression is regulated by transcription factors Pdr1p and Pdr3p. }\end{array}$ & 163.60 & 193.65 & $8.69 \times 10^{-1}$ \\
\hline HXT10 & $\begin{array}{l}\text { Putative hexose transporter, expressed at low levels and expression is repressed by } \\
\text { glucose. }\end{array}$ & 3.64 & 18.35 & $6.44 \times 10^{-1}$ \\
\hline HXT13 & $\begin{array}{l}\text { Hexose transporter, induced in the presence of non-fermentable carbon sources, induced } \\
\text { in low glucose and repressed in high glucose. }\end{array}$ & 124014.98 & 588.09 & $3.34 \times 10^{-7}$ \\
\hline HXT14 & $\begin{array}{l}\text { Protein similar to hexose transporter family members, expression is induced in low } \\
\text { glucose and repressed in high glucose }\end{array}$ & 9.70 & 12.23 & $9.70 \times 10^{-1}$ \\
\hline
\end{tabular}

\section{Other unique genes lead to unique phenotypes}

KHR1 is another unique gene of MT1, encoding a heatresistant killer toxin. This gene is located in a $2-\mathrm{kb}$ fragment inserted into MT1 scaffold 22, which can be mapped to $\mathrm{S} 288 \mathrm{c}$ chromosome IX. This ORF has been identified at the same location in the genome of YJM789 [31]. The killer toxin provides MT1 with a stronger survival rate compared with other microorganisms in the Chinese liquor fermentation environment. In addition, two specific $B I O$ genes were found on contig40, resulting in MT1 having biotin prototrophy. The two $B I O$ genes share $100 \%$ identity with BIO1 and BIO6, which encode pimeloyl-CoA synthase and KAPA synthase [32], respectively. They are also conserved (over $99 \%$ ) in several other S. cerevisiae strains, such as Sigma1278b (GenBank: ACVY00000000.1), YJM269 and UC5 (GenBank: AFDD00000000.1). As shown in Additional file 1: Figure $\mathrm{S} 2, \mathrm{BIO1}$ is located between ESMT1 and MCM10, while BIO6 lies between $A X L 2$ and REV7. Compared with S288c, MT1 could grow in the medium without biotin (data notshown). This confirmed the biotin prototroph of MT1.

\section{Conclusions}

The physiological and biochemical characteristic diversity of microbes is very important for industrial applications. Our analysis indicated that MT1 not only had higher tolerances to acidity and ethanol but was also capable of simultaneously producing ethanol from various carbon sources without glucose repression. These abilities could be applied to cellulose ethanol to eliminate the bottleneck during multi-carbon source fermentation. In addition, MT1 is a biotin prototroph, a phenotypic trait with potential in industrial applications. Biotin (vitamin $\mathrm{H}$ ) is an essential nutrient for all organisms, being a cofactor of many enzymes involved in carboxylation reactions, such as gluconeogenesis, amino acid metabolism, fatty acid biosynthesis and energy metabolism [33]. These physiological and biochemical characteristics place MT1 in a special category of yeast desirable for industry. Revealing the genetic basis of these traits would provide a platform for not only understanding Chinese liquor but also for developing an economically advanced biofuel process. It is necessary to analyze the evolutionary mechanisms of such special phenotypic and physiological characteristics. We assume that these variances may be induced by the raw materials and environmental stresses in the process of Chinese liquor-making. The SSF technique used in making Chinese liquor combines the enzymatic hydrolysis of starch with the simultaneous fermentation of sugars. Diverse hydrolases produced by the complex filamentous fungal community generate various sugars. This results in various carbohydrates that can be utilized by cells to grow and produce ethanol. In addition, Chinese Maotai-flavored liquor is produced by a complicated SSF process in an extremely severe environment, with high temperatures, as well as acidic and ethanol stresses. In the environment, temperatures could reach $50{ }^{\circ} \mathrm{C}$, the $\mathrm{pH}$ could be as low as 3.5 , and the ethanol concentration could be as high as $5.5 \%$. Over time, this specific environment has produced specific physiological properties and performances.

Environmental pressure is the external induction force, while the specific genome contains the internal underlying determinants. The genomic structural analysis indicated that MT1 retained a smaller genome than the reference strain S288c and some other industrial strains (Table 1). The genome of MT1 has lost several segments mostly comprised of non-coding protein genes, 
such as the large missing fragment of $25 \mathrm{~kb}$ on Chromosome I (Additional file 1: Figure S3A), which was verified by PCR (Additional file 1: Figure S3B). In S288c, this segment contains only one functional gene, FLO9. MT1 also lost hundreds of genes whose functions fell within transcription, translation, DNA and RNA binding, and cellular component synthesis. In addition, MT1 gained several specific genes, such as MEL1 and MAL63, related to its multi-carbon co-utilization, and $K H R 1$, associated with its competitiveness. These genomic adaptations embody the economy of the MT1 strain: removing some redundant genes, retaining necessary genes for growth and fermentation, and adapting to the complex fermentation environment of Chinese liquor. Consequently, these alterations make MT1 a naturally ascendant strain with a minimal genome, containing special functional genes/ clusters [34]. The small genome has a higher economic efficiency. It may have been beneficial to reduce the metabolic redundancy and then improve the metabolic efficiency [35]. Therefore, MT1 has the advantages of a short growth cycle, high production efficiency, high conversion rate, and strain stability in the fermentation process.

Various mechanisms are known to be involved in the adaptive evolution of yeast to the fermentation process, such as gene duplication, polyploidy, chromosomal rearrangements, interspecific hybridization and introgression [36]. On the basis of diverse examples drawn from both mammalian and microbial genetics, Maynard V. Olson proposed the "less is more" hypothesis that states that genetic loss may be an important engine of evolutionary change [37]. Aravind et al. [38] and Braun et al. [39] revealed that $S$. cerevisiae may have lost at least 300 genes, and another 300 or so genes have become highly diverged, since their split from their common ancestor with Saccharomyces pombe. A notable feature of the lost genes is the co-elimination of functionally connected groups of proteins, such as the signalosome and the spliceosome components. The other missing genes are involved in basic cellular processes, translation and ion homeostasis. However, intriguingly, some of the strains in which genes had been disrupted grew better than wildtype strains on a rich medium [40]. The counterintuitive idea that "less is more", is well-reflected in the evolution of MT1. The gene loss in MT1 has resulted in no obvious disadvantages in growth and fermentation, but provide MT1 the advantages of a short growth cycle and strain stability. Furthermore, a speculative hypothesis is proposed that MT1 gained the genomic space to obtain special functional genes (such as MEL1, MAL63, BIO1, BIO6 and KHR1) by reducing the gene redundancy. This strategy of evolution by gene loss is an important aspect of yeast diversification and may play a major role in their adaptation to the Chinese liquor fermentation ecosystem.
By revealing the genetic basis of specific phenotypes and elucidating the evolutionary history of the Chinese Maotai-flavored liquor yeast MT1, this work enriches our knowledge of S. cerevisiae, especially with regard to minimal genomic research. Furthermore, this functional genomics study of industrial microorganisms could be beneficial in improving breeding strategies to obtain the desired production traits in industrial microorganisms.

\section{Methods}

\section{Yeast strains and culture conditions}

S. cerevisiae S288c and sake yeast Kyokai No. 7 (K7) were obtained from the National Institute of Technology and Evaluation. Diploid strain MT1 was isolated from samples of the Maotai-flavored liquor production distillery in Guizhou, China. Strains from wine, beer or Chinese Rice Wine (Huangjiu) were obtained from China Center of Industrial Culture Collection (CICC). The yeast extract peptone dextrose (YPD) growth medium contained $10 \mathrm{~g} / \mathrm{L}$ yeast extract, $20 \mathrm{~g} / \mathrm{L}$ peptone and $20 \mathrm{~g} / \mathrm{L}$ glucose.

\section{Analysis of yeast tolerance to several stress conditions}

Three stress factors that are important features in liquor fermentation were studied: temperature, $\mathrm{pH}$, and ethanol concentration. Except for during the temperature stress test, all cultures were incubated at $30{ }^{\circ} \mathrm{C}$. For each stress factor, several fermentation conditions were tested, including four temperatures $\left(30,34,38\right.$ and $\left.42{ }^{\circ} \mathrm{C}\right)$, a range of $\mathrm{pH}$ levels (2.0 to 5.5) and several ethanol concentrations (0 to $16 \%$ ). Yeast was precultured in YPD medium at $30{ }^{\circ} \mathrm{C}$ for $24 \mathrm{~h}$. Cells were diluted in sterile water and then was cultured in stress media under static cultivation at $30{ }^{\circ} \mathrm{C}$ for $48 \mathrm{~h}$ with the same inoculum concentration of $1 \times 104 \mathrm{cfu} / \mathrm{mL}$.

\section{Carbon source assimilation experiment}

Biolog YT MicroPlates were used to test the ability of MT1 and S288c to utilize or oxidize compounds from a preselected panel of different carbon sources. The test yields a characteristic pattern of purple wells, which constitutes a "Metabolic Fingerprint". The isolate to be identified is grown on an agar medium and then suspended in sterile water. Then, the cell suspension is inoculated into the YT MicroPlate, at $100 \mu \mathrm{l}$ per well. All of the wells are colorless when inoculated. During the incubation there is a burst of respiration in the wells, which contain chemicals that can be oxidized, and the cells reduce the tetrazolium dye, forming a purple color. Meanwhile, microbes grow in the wells that contain utilizable carbon sources, leading to a higher turbidity. Negative wells remain colorless, as do the reference wells (A1 and D1) that contain no carbon sources. The $\mathrm{OD}_{590}$ values of lines $\mathrm{A}-\mathrm{C}$ and the $\mathrm{OD}_{730}$ values of lines $\mathrm{D}-\mathrm{H}$ were detected after $48 \mathrm{~h}$ of cultivation at $30{ }^{\circ} \mathrm{C}$. 
These values indicated that the carbon source could not be utilized when the corresponding $\mathrm{OD}_{590}$ or $\mathrm{OD}_{730}$ was below 0.2 , but could be at values from 0.2 to 0.8 , with values $>0.8$ having the best carbon utilization rates.

\section{Preparation of the sorghum extract medium}

To prepare the sorghum extract medium, $2 \mathrm{~kg}$ milled sorghum powder was added to $8 \mathrm{~L}$ deionized water. The mixture was steamed for $2 \mathrm{~h}$ and then saccharified by glucoamylase $(5 \mathrm{U} / \mathrm{L})$ at $60{ }^{\circ} \mathrm{C}$ for $4 \mathrm{~h}$. The supernatant was collected after being filtered through gauze and centrifuged at $8,000 \times g$ for $15 \mathrm{~min}$. The sugar content was measured on a Leica refractometer (Fisher Scientific, Pittsburg, PA, USA). Apply few solution on the scope then gently close the cover plate. Turn the eyepiece to read the value by adjusting the handwheel. The mixture was then diluted with water to produce a final sugar concentration of $10^{\circ}$ Brix $(80 \pm 5 \mathrm{~g} / \mathrm{L}$ reducing sugar) before sterilization.

\section{Sequencing and assembly}

The MT1 genome was sequenced using the wholegenome shotgun sequencing approach in the Illumina Miseq platform [41], and four paired-end/mate-paired sequencing libraries were constructed with insert sizes of $450 \mathrm{bp}, 700 \mathrm{bp}, 3 \mathrm{~kb}$ and $8 \mathrm{~kb}$. Raw data were assembled into contigs and scaffolds employing the de novo assembler Newbler [42] and SSPACE [43]. The assembled sequences were manually checked, and the gaps were closed using the GapCloser program [44]. The sequences of the final contigs were deposited in GenBank under the Whole Genome Shotgun project [GenBank: JRUS00000000].

\section{Gene prediction and annotation}

We predicted the MT1 genome using the MAKER pipeline prediction system, combining Augustus [45], SNAP [46] and the Glimmer gene prediction software [47]. The functional annotation was based on GO and KEGG [48] databases. Orthology with the S288c ORFs was evaluated by employing BLASTP similarities.

\section{Sequence comparison and SNPs/Indels analysis}

An overall comparison of the MT1 genome sequence with 16 chromosome sequences from S288c was performed using BLASTN. SNPs and Indels based on strain S288c were estimated using the GATK pipeline system and Unified Genotyper, and then they were annotated using Variant Effect Predictor provided by Ensembl.

\section{Construction of the evolutionary tree}

First, a family containing 18 yeast genomes was created using the software Orthomcl to obtain the protein sequences with a strict 1:1:1 ratio. Then, the protein sequences were compared using Muscle (version 3.8.31) software with the default parameters. Unreliable sequence alignment loci were removed using Gblock (version $0.91 \mathrm{~b}$ ). Finally, the phylogenetic tree was constructed by Mega5 software using the neighbor-joining method [18], Poisson model and uniform rates. We also used the bootstrap method to validate the reliability of the evolutionary tree branches with 1,000 bootstrap replications.

\section{CDNA preparation and transcriptomic analysis using RNA-seq}

Genomic DNA was digested using DNase, and total RNA was isolated using the TRIzol reagent. The OligoTex mRNA mini kit (Qiagen) was used to purify poly(A) mRNA from the total RNA samples. Then, the mRNA was fragmented using chemical reagents and high temperatures, and the DNA was synthesized, purified and enriched. The sequencing of the cDNA library products was performed on an Illumina HiSeq 2000 platform. After removing reads containing sequencing adapters and reads of low quality [reads in which the percentage of low quality bases (quality value $\leq 5$ ) was more than $50 \%$ ], the remaining clear reads were aligned to $S$. cerevisiae S288c or MT1 genes using bowtie2/tophat2. The expression levels were normalized by reads per kilobase of exon region per million mapped reads (RPKM) [49]. The screening of differentially expressed genes and $P$-value calculations were performed with the method of DESeq [50]. Three biological replicates were performed for each time point for each strain.

\section{Additional file}

Additional file 1: Table S1. Statistics of the SNPS and Indels among the MT1 Chromosomes. Table S2. Annotation of SNPs. Table S3. Annotation of Indels. Table S4. Copy Number Variations (CNVs) between MT1 and S288c. Table S5. Functions of the missed genes. Figure S1. Statistical analysis on the number of contigs with identity $<95 \%$ in the amino acid levels. Figure S2. Analysis of two BIO genes present in the $\mathrm{LI}$ genome, but not in the $5288 \mathrm{C}$ genome. A: Schematic organization of two relevant segments from MT1-contig40, S288c-Chr.IX and K7-Chr.IX; B: Dot Matrix Comparison maps of the DNA sequences between BIO1-MT1/BIO6-MT1 and BIO1-Ref/BIO6-Ref; C: Dot Matrix Comparison maps of the protein sequences between BIO1-MT1/BIO6-MT1 and B/O1-Ref/BIO6-Ref. Figure S3. A: Colinearity analysis of Chr. I. The same Locally Collinear Block is represented by same color and vertical lines represent the same LCB; The height of internal vertical lines in LCB on behalf of the level of sequence consistency; Blank in one strain stands for the region does not exist in the other. B: PCR profiles of the $25 \mathrm{~Kb}$ fragment specific to $5288 \mathrm{C}$ which was divided into 4 small segments (S1, S2, S3, S4). In addition, 265 rDNA gene was detected as the positive control. M: DNA maker of 5000 bp. (DOC 849 kb)

\section{Competing interests}

All authors (LXW, WQ, ZY and $X Y$ ) declare that we have no competing interests.

\section{Authors' contributions}

LXW, WQ and ZY drafted the manuscript. LXW and WQ performed the physiological analysis and genome sequencing and molecular genetic 
studies. WQ and XY participated in the design of the study. All authors read and approved the final manuscript.

\section{Acknowledgments}

This work was supported by the National High Technology Research and Development Program of China (2013AA102108 and 2012AA021301); National Natural Science Foundation of China (31371822 and 31271921); China Postdoctoral Science Foundation (2014 M550265); Priority Academic Program Development of Jiangsu Higher Education Institutions, the 111 Project (No. 111-2-06); and the Jiangsu Province "Collaborative Innovation Center for Advanced Industrial Fermentation" industry development program.

\section{Author details}

${ }^{1}$ State Key Laboratory of Food Science and Technology; The Key Laboratory of Industrial Biotechnology, Ministry of Education; Synergetic Innovation Center of Food Safety and Nutrition; School of Biotechnology, Jiangnan University, Wuxi, Jiangsu, China. ${ }^{2}$ Ministry of Education Key Laboratory of Systems Biomedicine, Shanghai Center for Systems Biomedicine, Shanghai Jiao Tong University, Shanghai, China.

Received: 16 May 2015 Accepted: 30 November 2015

\section{Published online: 15 December 2015}

\section{References}

1. Zhu S, Lu X, Ji K, Guo K, Li Y, Wu C, et al. Characterization of flavor compounds in Chinese liquor Moutai by comprehensive two-dimensional gas chromatography/time-of-flight mass spectrometry. Anal Chim Acta. 2007:597(2):340-8.

2. Ballesteros M, Oliva JM, Negro MJ, Manzanares P, Ballesteros I. Ethanol from lignocellulosic materials by a simultaneous saccharification and fermentation process (SFS) with Kluyveromyces marxianus CECT 10875. Process Biochem. 2004;39(12):1843-8.

3. Li X-R, Ma E-B, Yan L-Z, Meng H, Du X-W, Zhang S-W, et al. Bacterial and fungal diversity in the traditional Chinese liquor fermentation process. Int $J$ Food Microbiol. 2011;146(1):31-7.

4. Chen $B, W u Q, X u Y$. Filamentous fungal diversity and community structure associated with the solid state fermentation of Chinese Maotai-flavor liquor. Int J Food Microbiol. 2014;179:80-4.

5. Pandey A. Solid-state fermentation. Biochem Eng J. 2003;13(2):81-4.

6. Xu Y, Ji K Moutai (Maotai): production and sensory properties. Alcoholic beverages: sensory evaluation and consumer research. 2012;315-30.

7. Wang C-I, D-j S, G-I G. Microorganisms in Daqu: a starter culture of Chinese Maotai-flavor liquor. World J Microbiol Biotechnol. 2008;24(10):2183-90.

8. Wu Q, Xu Y, Chen L. Diversity of yeast species during fermentative process contributing to Chinese Maotai-flavour liquor making. Lett Appl Microbiol. 2012;55(4):301-7.

9. Xu Y, Ji K, Piggott J. Moutai (Maotai): production and sensory properties. Alcoholic Beverages: Sensory Evaluation and Consumer Research. 2012;315-30.

10. Goffeau A, Barrell B, Bussey H, Davis R, Dujon B, Feldmann H, et al. Life with 6000 genes. Science. 1996;274(5287):546-67.

11. Kumar A, Snyder M. Emerging technologies in yeast genomics. Nat Rev Genet. 2001;2(4):302-12.

12. Ralser M, Kuhl H, Ralser M, Werber M, Lehrach $H$, Breitenbach $M$, et al. The Saccharomyces cerevisiae W303-K6001 cross-platform genome sequence: insights into ancestry and physiology of a laboratory mutt. Open bio. 2012;2(8):120093.

13. Akao T, Yashiro I, Hosoyama A, Kitagaki H, Horikawa H, Watanabe D, et al. Whole-genome sequencing of sake yeast Saccharomyces cerevisiae Kyokai no. 7. DNA Res. 2011;18(6):423-34

14. Borneman AR, Desany BA, Riches D, Affourtit JP, Forgan AH, Pretorius IS, et al. Whole-genome comparison reveals novel genetic elements that characterize the genome of industrial strains of Saccharomyces cerevisiae. PLoS Genet. 2011;7(2):e1001287.

15. Borneman AR, Forgan AH, Pretorius IS, Chambers PJ. Comparative genome analysis of a Saccharomyces cerevisiae wine strain. FEMS Yeast Res. 2008;8(7):1185-95.

16. Novo M, Bigey F, Beyne E, Galeote V, Gavory F, Mallet S, et al. Eukaryote-toeukaryote gene transfer events revealed by the genome sequence of the wine yeast Saccharomyces cerevisiae EC1118. Proc Natl Acad Sci U S A. 2009;106(38):16333-8.
17. Argueso JL, Carazzolle MF, Mieczkowski PA, Duarte FM, Netto OV, Missawa SK, et al. Genome structure of a Saccharomyces cerevisiae strain widely used in bioethanol production. Genome Res. 2009;19(12):2258-70.

18. Liti G, Carter DM, Moses AM, Warringer J, Parts L, James SA, et al. Population genomics of domestic and wild yeasts. Nature. 2009;458(7236):337-41.

19. Zheng D-Q, Wang P-M, Chen J, Zhang K, Liu T-Z, Wu X-C, et al. Genome sequencing and genetic breeding of a bioethanol Saccharomyces cerevisiae strain YJS329. BMC Genomics. 2012;13(1):479.

20. Dowell RD, Ryan O, Jansen A, Cheung D, Agarwala S, Danford T, et al. Genotype to phenotype: a complex problem. Science. 2010;328(5977):469.

21. Jenkins GM, Richards A, Wahl T, Mao C, Obeid L, Hannun Y. Involvement of yeast sphingolipids in the heat stress response of Saccharomyces cerevisiae. J Biol Chem. 1997;272(51):32566-72.

22. Kawahata M, Masaki K, Fujii T, lefuji H. Yeast genes involved in response to lactic acid and acetic acid: acidic conditions caused by the organic acids in Saccharomyces cerevisiae cultures induce expression of intracellular metal metabolism genes regulated by Aft1p. FEMS Yeast Res. 2006;6(6):924-36.

23. Barnett JA. The utilization of sugars by yeasts. Adv Carbohydr Chem Biochem. 1975;32:125-234.

24. Abyzov A, Urban AE, Snyder M, Gerstein M. CNVnator: an approach to discover, genotype, and characterize typical and atypical CNVs from family and population genome sequencing. Genome Res. 2011;21(6):974-84.

25. Platt A, Reece RJ. The yeast galactose genetic switch is mediated by the formation of a Gal4p-Gal80p-Gal3p complex. EMBO J. 1998;17(14):4086-91.

26. Nijkamp JF, van den Broek M, Datema E, de Kok S, Bosman L, Luttik MA, et al. De novo sequencing, assembly and analysis of the genome of the laboratory strain Saccharomyces cerevisiae CEN.PK113-7D, a model for modern industrial biotechnology. Microb Cell Factories. 2012;11:36.

27. Daran-Lapujade P, Jansen ML, Daran JM, van Gulik W, de Winde JH, Pronk $J$ T. Role of transcriptional regulation in controlling fluxes in central carbon metabolism of Saccharomyces cerevisiae. A chemostat culture study. J Biol Chem. 2004;279(10):9125-38.

28. Chow TH, Sollitti P, Marmur J. Structure of the multigene family of MAL loc in Saccharomyces. Mol Gen Genet. 1989;217(1):60-9.

29. Kruckeberg AL. The hexose transporter family of Saccharomyces cerevisiae Arch Microbiol. 1996;166(5):283-92.

30. Greatrix BW, van Vuuren HJ. Expression of the HXT13, HXT15 and HXT17 genes in Saccharomyces cerevisiae and stabilization of the HXT1 gene transcript by sugar-induced osmotic stress. Curr Genet. 2006;49(4): 205-17.

31. Wei W, McCusker JH, Hyman RW, Jones T, Ning Y, Cao Z, et al. Genome sequencing and comparative analysis of Saccharomyces cerevisiae strain YJM789. Proc Natl Acad Sci U S A. 2007;104(31):12825-30.

32. $\mathrm{Wu} \mathrm{H}$, Ito $\mathrm{K}$, Shimoi $\mathrm{H}$. Identification and characterization of a novel biotin biosynthesis gene in Saccharomyces cerevisiae. Appl Environ Microbiol. 2005;71(11):6845-55.

33. Ohsugi M, Imanishi Y. Microbiological activity of biotin-vitamers. J Nutr Sci Vitaminol. 1985;31(6):563-72.

34. Koonin EV. How many genes can make a cell: The minimal-gene-Set concept 1. Annu Rev Genomics Hum Genet. 2000;1(1):99-116.

35. Murakami K, Tao E, Ito Y, Sugiyama M, Kaneko Y, Harashima S, et al. Large scale deletions in the Saccharomyces cerevisiae genome create strains with altered regulation of carbon metabolism. Appl Microbiol Biotechnol. 2007;75(3):589-97.

36. Liti G, Louis EJ. Yeast evolution and comparative genomics. Annu Rev Microbiol. 2005:59:135-53.

37. Olson MV. When less is more: gene loss as an engine of evolutionary change. Am J Hum Genet. 1999;64(1):18-23.

38. Aravind L, Watanabe H, Lipman DJ, Koonin EV. Lineage-specific loss and divergence of functionally linked genes in eukaryotes. Proc Natl Acad Sci. 2000;97(21):11319-24.

39. Braun EL. Large-scale comparison of fungal sequence information: Mechanisms of innovation in Neurospora crassa and gene loss in saccharomyces cerevisiae. Genome Res. 2000;10(4):416-30.

40. Goebl MG, Petes TD. Most of the yeast genomic sequences are not essential for cell growth and division. Cell. 1986;46(7):983-92.

41. Quail MA, Smith M, Coupland P, Otto TD, Harris SR, Connor TR, et al. A tale of three next generation sequencing platforms: comparison of Ion Torrent Pacific Biosciences and Illumina MiSeq sequencers. BMC Genomics. 2012;13:341. 
42. Nederbragt AJ. On the middle ground between open source and commercial software-the case of the Newbler program. Genome Biol. 2014;15(4):113

43. Boetzer M, Henkel CV, Jansen HJ, Butler D, Pirovano W. Scaffolding pre-assembled contigs using SSPACE. Bioinformatics. 2010;27(4):578-9.

44. Boetzer M, Pirovano W. Toward almost closed genomes with GapFiller Genome Biol. 2012;13(6):R56.

45. Stanke M, Waack S. Gene prediction with a hidden Markov model and a new intron submodel. Bioinformatics. 2003;19 Suppl 2:ii215-25.

46. Korf I. Gene finding in novel genomes. BMC bioinformatics. 2004;5(1):59.

47. Delcher AL, Harmon D, Kasif S, White O, Salzberg SL. Improved microbial gene identification with GLIMMER. Nucleic Acids Res. 1999;27(23):4636-41.

48. Kanehisa M, Goto S, Sato Y, Furumichi M, Tanabe M. KEGG for integration and interpretation of large-scale molecular data sets. Nucleic Acids Res. 2012:40(Database issue):D109-114.

49. Mortazavi A, Williams BA, McCue K, Schaeffer L, Wold B. Mapping and quantifying mammalian transcriptomes by RNA-Seq. Nat Methods. 2008:5(7):621-8,

50. Anders S, Huber W. Differential expression of RNA-Seq data at the gene level-the DESeq package. Heidelberg: European Molecular Biology Laboratory (EMBL); 2012

51. Tamura K, Peterson D, Peterson N, Stecher G, Nei M, Kumar S. MEGA5: molecular evolutionary genetics analysis using maximum likelihood, evolutionary distance, and maximum parsimony methods. Mol Biol Evol. 2011;28(10):2731-9.

\section{Submit your next manuscript to BioMed Central and we will help you at every step:}

- We accept pre-submission inquiries

- Our selector tool helps you to find the most relevant journal

- We provide round the clock customer support

- Convenient online submission

- Thorough peer review

- Inclusion in PubMed and all major indexing services

- Maximum visibility for your research 